\title{
EDUCAR EN MEDIOS SOCIALMENTE VULNERABLES: EL VALOR DE LA CAPACITACIÓN DOCENTE
}

\author{
Laura Beatriz Oros \\ IDIC-CONICET; Instituto Superior \\ Adventista de Misiones (Argentina) \\ María Soledad Menghi \\ CIIPME-CONICET; Universidad Católica \\ Argentina, Sede Paraná (Argentina) \\ María Cristina Richaud \\ CONICET (Argentina) \\ María Ghiglione \\ Universidad Católica Argentina, \\ Sede Paraná (Argentina)
}

RESUMEN: Los maestros que trabajan con poblaciones marginales necesitan conocimientos y estrategias psicopedagógicas específicas para impulsar de forma eficaz la promoción del aprendizaje en este grupo de niños. Tomando como base este supuesto, se desarrolló una propuesta de capacitación docente para aumentar los conocimientos del maestro sobre las necesidades sociales y afectivas que tienen los niños en riesgo, y para perfeccionarlo en la implementación de acciones concretas que fortalecen los recursos psicosociales y optimizan el aprendizaje. El propósito de este artículo es realizar un análisis descriptivo de las opiniones docentes respecto al valor de utilidad de la capacitación implementada. Este estudio se desarrolló con una muestra de 83 docentes de los niveles inicial y primario que participaron de las instancias de perfeccionamiento llevadas a cabo en las provincias de Entre Ríos, Santa Fe, y Buenos Aires, Argentina. Se utilizó un Cuestionario de Valoración elaborado ad hoc $(a=0,96)$, a través del cual se encontraron evidencias favorables acerca del valor de la implementación de estas instancias de capacitación docente en el ejercicio de la docencia en medios socialmente vulnerables.

PALABRAS CLAVE: Profesión docente, programa de formación, investigación empírica, vulnerabilidad social. 


\title{
EDUCATING IN SOCIALLY VULNERABLE ENVIRONMENTS: THE VALUE OF TEACHER'S TRAINING
}

\begin{abstract}
Assuming that teachers who work with marginalized populations need appropriate knowledge and strategies based on educational psychology to promote, effectively, learning in this group of children, a teacher's training proposal was developed. It was based on the following objectives: (a) increase teachers knowledge about social and emotional needs in children at risk; (b) generate awareness of the importance of attending this needs in the learning process and in the consolidation of the knowledge proposed by the designed curriculum; (c) instruct teachers in the implementation of concrete actions to strengthen the resources that optimize learning; and (d) train teachers as multiplier agents to transfer to the rest of the educative staff this acquired knowledge and practices. The purpose of this paper is a descriptive analysis of the teachers' opinions about the utility value of the implemented training. This study was developed with a sample of 83 teachers of the kindergarten and primary levels, who participated in the training, executed in the provinces of Entre Rios, Santa Fe and Buenos Aires, Argentina. An Assessment Questionnaire developed ad hoc $(\mathrm{a}=0.96)$ was used, whereby was found favorable evidence about the value of implementing these instances of teacher's training in the practice of teaching in socially vulnerable enviroments.
\end{abstract}

KEYWORDS: Teaching profession, training programme, empirical research, social vulnerability.

Recibido: 13/06/2014

Aceptado: 09/11/2014

\section{INTRODUCCIÓN}

Para la gran mayoría de los niños, la pobreza extrema implica riesgos en su desarrollo ya que se asocia con retraso de crecimiento fetal, bajo peso al nacer, mortalidad infantil, mayor probabilidad de enfermedades dermatológicas, respiratorias e intestinales, y menor organización cerebral (Paredes, 2013; Bolzán y Mercer, 2009; Richaud de Minzi et al., 2013; Solano, Acuña, Barón, Morón de Salim y Sánchez, 2008).

Muchos de estos niños crecen en barrios con altas tasas de desempleo y sin los mínimos cuidados para un desarrollo saludable. Entre otros problemas, predominan la ruptura familiar, la conducta antisocial, las redes sociales restringidas a los límites del entorno marginal y la falta de control social informal sobre las conductas y actividades de niños y adultos en el barrio (Wilson, 1996).

Por otra parte, se sabe que los niños en situación de vulnerabilidad social por pobreza suelen presentar altas tasas de problemas socioemocionales y de conducta, incluyendo ansiedad, aislamiento social, agresión, distrés psicológico y baja autoestima y autoeficacia (Lupien, King, Meaney, y McEwen, 2001; McLoyd y Wilson, 1991). 
Con respecto al plano cognitivo, es habitual que presenten atrasos en el desarrollo intelectual y en los logros escolares (especialmente relacionados a limitaciones lingüísticas, de memoria y atención) (Arán Filippetti y Richaud de Minzi, 2011). Cuando comienzan la educación preescolar tienden a tener menores niveles de habilidades relacionadas con la escuela que los niños no pobres; su progreso en la escuela es más lento y muchos terminan por desertar (McLanahan, Astone, y Marks, 1991; Ramey y Campbell, 1991).

Entre las razones que dan cuenta de este fracaso, juegan un papel relevante las diferencias lingüísticas y culturales entre los entornos en los que crecen los niños y las escuelas a las que asisten. Hay evidencia de que los niños en riesgo ambiental tienen necesidades especiales basadas en diferencias biológicas y sociales.

Entre los factores biológicos que afectan la cognición y la conducta están la exposición a la contaminación de diferentes tipos, al alcohol y al tabaco durante el embarazo, bajo peso al nacer y deficiencias en micronutrientes, en particular el hierro (Lozoff, Jimenez, Hagen, Mollen, y Wolf, 2000; Milman, 2012; Singer, Minnes, Short, Arendt, Farkas, Lewis, Klein, Russ, Min y Kirchner, 2004; Stanco, 2007).

Además de una mayor exposición a factores de riesgo biológicos, existen factores de riesgo sociales asociados al tipo de ambiente en el hogar. Se ha demostrado que los adultos que están inmersos en situación crónica de pobreza tienen mayor probabilidad de presentar depresión, ansiedad y fatalismo; una baja respuesta a las necesidades de los niños, escasa estimulación del lenguaje, violencia familiar, y una pobre calidad de los cuidados disponibles (Ardila, 2012; Bradley et al., 1994; Cidade, Moura y Ximenes, 2012; Galindo y Ardila, 2012; Garret, Ng'andu y Ferron, 1994; Smith y Sandhu, 2004; Patel y Kleinman, 2003). Aunque la pobreza y la vulnerabilidad social no son lo mismo, es claro que los niños pobres tienen un alto riesgo de volverse socialmente vulnerables (Richaud de Minzi, 2013).

La vulnerabilidad social implica un proceso de desarrollo humano donde el niño está expuesto a un deterioro psicofísico por privaciones tales como la alimentación, afecto, protección, educación, entre otras. Se relaciona con niños que han sido gestados, criados o socializados, expuestos a una serie de factores que han ido restringiendo gradualmente sus posibilidades de desarrollo individual y de integración social (Richaud et al., 2013: 422).

La incidencia de la pobreza en Latinoamérica entre los niños menores de 15 años es en promedio, 1,7 veces mayor a la de las personas que superan esa edad. En la mayoría de los países, entre 1990 y 2008, se produjo un aumento significativo del cociente entre las tasas de pobreza de los menores y los adultos, especialmente en Argentina, Brasil, Panamá, Uruguay y Venezuela (CEPAL, 2009). Puntualmente en Argentina, más del $40 \%$ de la población pobre corresponde a niños menores de 15 años de edad (Gasparini y Cruces, 2010), traduciéndose los efectos de la pobreza en un incremento preocupante de la desnutrición, la mortalidad infantil, y los problemas escolares, como los aplazos, la deserción y la repitencia (Lacunza, 2010).

La escuela, preparada para un niño estándar, supone en su currículo que todos los niños han alcanzado el desarrollo esperable en cada edad, y propone actividades acordes a ese desarrollo. Por ello, maestros no capacitados para hacer frente a las demandas diferentes de los niños en riesgo, tratan de ir adelante en el cumpli- 
miento de un currículo que no tiene en cuenta esta diversidad y terminan sumiendo a estos niños en una nueva tragedia en sus vidas, el fracaso escolar. Fracaso que también es vivido por los docentes como propio, aumentando así su desánimo, frustración y baja realización laboral.

Esto reclama que los programas de formación de maestros tengan en cuenta la problemática del niño en riesgo ambiental, y les aporten el conocimiento y entrenamiento adecuado para manejarse y enseñar a alumnos con estas características, y a manejar al mismo tiempo las complejas relaciones con los padres y el entorno social de pobreza, en general. La falta de herramientas para abordar esta problemática lleva a conflictos institucionales, sentimientos de indefensión en los docentes implicados, disminución de la autoconfianza, malestar y luego indiferencia frente al fracaso reiterado de los niños, enfrentamientos con las familias con las cuales no comparten marcos culturales, etc., todo lo cual conspira contra la salud física y mental de los docentes y el éxito en el desarrollo y aprendizaje de los niños (Henderson y Milstein, 2003).

Partiendo del supuesto de que los maestros que trabajan con poblaciones marginales necesitan conocimientos, estrategias pedagógicas, psicológicas y metodológicas apropiadas para impulsar en forma eficaz la promoción del aprendizaje en este grupo de niños, se desarrolló una propuesta de capacitación docente basada en los siguientes objetivos: (a) aumentar los conocimientos del docente sobre las necesidades sociales y afectivas que tienen los niños en riesgo; (b) generar concientización sobre la importancia de atender estas necesidades para que sea posible el aprendizaje y la consolidación de los conocimientos que propone el diseño curricular; (c) capacitar al docente en la implementación de acciones concretas para fortalecer los recursos cognitivos, sociales y afectivos que optimizan el aprendizaje; y (d) formar al docente como agente multiplicador para que transfiera al resto del plantel educativo, los conocimientos y las prácticas adquiridos.

Esta propuesta fue concretada en el marco del Programa "Sin afecto no se aprende ni se crece. Un Programa para reforzar los recursos afectivos, cognitivos y lingüísticos en niños en riesgo por pobreza extrema", que se llevó a cabo por investigadores del Centro Interdisciplinario de Investigaciones en Psicología Matemática y Experimental (CIIPME), dependiente del CONICET (Richaud de Minzi, 2009).

Si bien en nuestro país ha habido algunas iniciativas interesantes de postítulos que incluyen como tema central la educación infantil en contextos de vulnerabilidad social, no se conoce la eficacia de dichas instancias, la utilidad concreta de su aplicación a la realidad cotidiana de los alumnos y docentes, ni la opinión que de ellos tienen los actores principales. El propósito de este artículo es realizar un análisis descriptivo de las opiniones docentes respecto al valor de utilidad de la capacitación implementada. La eficacia de esta propuesta, en términos de cambios cuantitativos y cualitativos en el desempeño y desarrollo de los niños y maestros, ha sido informada en estudios anteriores (ver Conclusiones). Analizamos ahora la mirada del docente sobre la utilidad de este programa de capacitación considerando que su valoración subjetiva puede aportar un elemento complementario de gran valor para juzgar la importancia y conveniencia de esta instancia de formación profesional. 


\section{Características de la CAPAcitación}

La capacitación fue diseñada para ser ofrecida a docentes de nivel inicial y primario que estuvieran ejerciendo su función en escuelas argentinas, urbano-marginales, incluidas en el Plan Nacional de Mil Escuelas Bajo el Nivel de Pobreza, gestionado del Ministerio de Educación de la República Argentina. Los establecimientos educativos que se cuentan dentro de este plan fueron seleccionados según criterios y prioridades fijados por el Consejo Federal de Cultura y Educación, en base a la situación socioeconómica de los alumnos y el índice de ausentismo. Por otra parte, y de acuerdo a la Escala Graffar (adaptación de Mendez Castellano, 1982), cabe mencionar que los alumnos que asisten a estas escuelas se ubican en los estratos IV y $\vee$ (pobreza relativa y pobreza crítica), de entre cinco estratos posibles que vienen definidos por: (a) la ocupación del jefe de familia, (b) la instrucción de la madre, (c) la principal fuente de ingreso familiar y (d) la condición habitacional.

El plan de capacitación propuesto combinó la modalidad intensiva con la modalidad continua. De acuerdo a Huberman (2005) la capacitación intensiva debe plantear "actividades en un corto período de tiempo, pero con sesiones de trabajo prolongadas. Exige del capacitador una preparación detallada, minuciosa y previa, y de los participantes, un alto nivel de motivación inicial para que su participación sea comprometida" (111-112). Siguiendo este esquema, se realizaron ocho talleres presenciales, de media jornada diaria, con periodicidad mensual. Se combinó la modalidad expositiva con actividades grupales y de discusión. Cada taller estuvo coordinado por un especialista diferente, experto en la temática a abordar. Participaron docentes, directivos y otros profesionales afectados al ámbito educativo. Al final de cada taller, se entregó a los participantes una guía de actividades prácticas para ser implementadas durante un plazo de aproximadamente 30 días. Culminados los ocho talleres, se realizó una evaluación final integradora.

La capacitación intensiva propuso al docente conocer, analizar y entrenarse para el fortalecimiento de recursos cognitivos, afectivos, sociales y lingüísticos que fomentan la resiliencia infantil. El primer encuentro fue introductorio y desarrolló el tema de la vulnerabilidad infantil asociada a la pobreza. Se explicaron los factores de riesgo psicosocial y su impacto sobre los procesos cognitivos, sociales y afectivos durante la niñez. En cada uno de los módulos subsiguientes se abordó una fortaleza psicosocial diferente: el apego, las relaciones positivas con los padres, las funciones ejecutivas, las capacidades lingüísticas, las emociones positivas, las habilidades sociales, el comportamiento prosocial y el afrontamiento al estrés. Cada concepto fue definido teóricamente y ejemplificado mediante la presentación de casos y situaciones cotidianas. Fueron presentados y discutidos algunos de sus factores desencadenantes y de sus consecuencias y beneficios sobre la salud general y el desarrollo académico de los niños. Se sugirieron algunas estrategias prácticas para promover y fortalecer cada uno de estos recursos dentro del aula, y mediante dinámicas grupales se estimuló a los docentes a que propusieran diferentes métodos para conseguir estos objetivos según su propia experiencia profesional.

Adicionalmente, formó parte de nuestras expectativas que los docentes realizaran un acercamiento a las herramientas tecnológicas aplicadas a la educación. Para 
ello dispusimos una plataforma virtual que ofreció la posibilidad a los participantes de optimizar tiempo, esfuerzo y recursos. Algunas de las prestaciones de este medio permitieron que el docente: (a) recibiera información actualizada sobre aspectos que hacían a la organización de los encuentros; (b) recibiera material bibliográfico en formato digital, y por lo tanto sin costo; (c) consultase su porcentaje de asistencia y la fecha de entrega de requisitos; (d) subiera a la web sus trabajos prácticos; (e) viera la calificación de sus trabajos y exámenes; (f) participara de un foro para compartir experiencias con el resto de los participantes; y (g) mantuviera un vínculo más fluido con los docentes del curso y el grupo de asistentes que disponían de horarios para la consulta on-line.

Los participantes recibieron diversos materiales de lectura obligatoria y documentos de apoyo. Muchos de éstos fueron elaborados expresamente para esta instancia y presentaron un formato didáctico y de fácil manejo

Por otra parte, la capacitación continua se desarrolló como una actividad académica organizada, dirigida específicamente a aquellos docentes que habían decidido implementar el programa en sus clases. Esta instancia de capacitación tuvo aplicabilidad inmediata y se realizó con el objeto de brindar asesoramiento personalizado para la integración del desarrollo socioafectivo al currículo escolar (para un ejemplo de este tipo de actividades puede consultarse Oros, Manucci y Richaud de Minzi, 2011).

Durante un período lectivo anual, un equipo de psicólogos y psicopedagogos trabajó con las maestras un día a la semana, durante tres horas, para diseñar la planificación escolar semanal (objetivos de la clase, contenidos curriculares a dictar, estrategias y técnicas didácticas para incluir la promoción de los recursos psicológicos junto a los contenidos curriculares, evaluaciones, tareas para la casa, etc.). El resto de los días, dentro del aula, se realizó la aplicación de estas actividades. Siempre que fue posible, un observador externo llevó un cuidadoso registro de las acciones realizadas por los miembros del equipo y los docentes, detallando asimismo las respuestas exhibidas por los niños, lo que permitió determinar el grado de éxito de las estrategias implementadas y evaluar el desempeño del maestro, decidiendo cambios si eran necesarios.

Esta última forma de capacitación garantizó una mayor participación y libertad de opinión del equipo docente, y la posibilidad de realizar eventuales modificaciones al plan de actividades bajo la supervisión de expertos.

\section{MÉTOdo}

\section{Sujetos}

Participaron de este estudio los 83 docentes que asistieron a las capacitaciones llevadas a cabo en las provincias de Entre Ríos, Santa Fe, y Buenos Aires, en la República Argentina. Los participantes estaban afectados al ámbito educativo de los niveles inicial y primario de diferentes escuelas de gestión pública y privada. Su valoración acerca de la utilidad del programa de capacitación, fue voluntaria y anónima. 


\section{Instrumento}

Los participantes completaron un Cuestionario de Valoración elaborado ad hoc con el objeto de expresar su opinión respecto al grado de valor y utilidad de esta formación teórico-práctica (Oros, Ghiglione y Menghi, 2010). El instrumento incluye 14 ítemes que se distribuyen factorialmente en 2 dimensiones que explican el $76,8 \%$ de la variancia: "Conocimientos y actitudes" $(a=0,96)$ y "Aplicación práctica y apreciación general" $(\mathrm{a}=0,91)$. A cada ítem le corresponden cinco opciones de respuesta según el grado de acuerdo que el docente manifiesta con el contenido de la frase. El cuestionario completo presenta una excelente consistencia interna $(a=0,96)$.

\section{Procedimientos de análisis de datos}

Los docentes completaron el cuestionario en forma colectiva, en el marco del último módulo de capacitación presencial. Los datos fueron tabulados y procesados mediante el programa estadístico SPSS. Para analizar descriptivamente las respuestas de los docentes, se calcularon frecuencias, porcentajes, medias y desvíos estándar.

\section{REsultados}

Los resultados respecto al cumplimiento de los primeros dos objetivos del programa de capacitación: (a) aumentar los conocimientos del docente sobre las necesidades sociales y afectivas que tienen los niños en riesgo; y (b) concientizarlo sobre la importancia de atender estas necesidades para que sea posible el aprendizaje y la consolidación de los conocimientos que propone el diseño curricular; fueron alentadores.

Más del $80 \%$ de los docentes afirmó que la capacitación aumentó su interés sobre el tema (86,6\%), incrementó sus conocimientos sobre las necesidades de los niños en riesgo y sus familias $(80,7 \%)$; amplió sus conocimientos sobre las fortalezas de los niños en riesgo $(83,1 \%)$, los ayudó a completar su formación académica (84\%), los motivó a incluir la promoción de recursos en la planificación curricular $(85,5 \%)$, y cubrió sus expectativas respecto a los objetivos inicialmente planteados $(81,3 \%)$ (Ver Tabla 1$)$.

Con respecto al objetivo 3: Capacitar a los docentes en la implementación de acciones concretas para fortalecer los recursos cognitivos, sociales y afectivos que optimizan el aprendizaje de estos niños; también se encontraron resultados satisfactorios. El 84,3\% expresó haber recibido entrenamiento relevante a las necesidades sociales, emocionales, de salud y educación de los niños en pobreza; el 71,1\% afirmó haber recibido herramientas concretas para distinguir cuáles son los niños con problemas asociados a la pobreza en sus clases; el 81,7\% manifestó haber realizado actividades prácticas que le sirvieron para apoyar y aclarar las exposiciones teóricas; más del $85 \%$ expresó que el material bibliográfico utilizado abordaba los temas en profundidad $(85,5 \%)$ y resultaba ameno para público no versado en investigación científica (91,5\%); el 84,3\% mencionó que los disertantes explicaron el programa en forma clara; el 91,5\% expresó que se aportaron ejemplos útiles y pertinentes a la función áulica cotidiana; y el 83,1\% sostuvo que el tiempo estuvo bien distribuido entre los diferentes contenidos teórico-prácticos (Ver Tabla 1). 
Tabla 1. Porcentajes, medias y desvíos estándar de las respuestas a los ítemes del Cuestionario de Valoración

\begin{tabular}{|c|c|c|c|c|c|c|c|}
\hline Afirmaciones & TA & A & $\mathbf{N}$ & D & TD & $M$ & $D E$ \\
\hline $\begin{array}{l}\text { 1. Aumentó mis conocimientos sobre las necesidades de } \\
\text { los niños en riesgo y sus familias }\end{array}$ & 36.4 & 31.6 & 12.1 & 3 & 1 & 4.18 & .90 \\
\hline $\begin{array}{l}\text { 2. Amplió mis conocimientos sobre las fortalezas de los } \\
\text { niños en riesgo }\end{array}$ & 35.4 & 34.3 & 11.1 & 1 & 2 & 4.19 & .89 \\
\hline 3. Ayudó a completar mi formación académica & 43.2 & 40.7 & 9.9 & 2.5 & 3.7 & 4.17 & .97 \\
\hline 4. Aumentó mi interés en el tema & 53.7 & 32.9 & 9.8 & 0 & 3.7 & 4.33 & .93 \\
\hline $\begin{array}{l}\text { 5. Me motivó a incluir la promoción de recurso } \\
\text { planificación curricular }\end{array}$ & 32.5 & 53 & 10.8 & 1.2 & 2.4 & 4.12 & .83 \\
\hline 6. Cubrió mis expectativas & 36.3 & 45 & 11.3 & 5 & 2.5 & 4.07 & .95 \\
\hline $\begin{array}{l}\text { 7. Proveyó entrenamiento relevante a las necesidades } \\
\text { sociales, emocionales, de salud y educación de los } \\
\text { niños en riesgo por pobreza }\end{array}$ & 37.3 & 47 & 12 & 2.4 & 1.2 & 4.17 & .82 \\
\hline $\begin{array}{l}\text { 8. Brindó herramientas concretas para distinguir cuáles } \\
\text { son los niños con problemas asociados a la pobreza } \\
\text { en sus clases }\end{array}$ & 28.9 & 42.2 & 24.1 & 4.5 & 0 & 3.95 & .85 \\
\hline $\begin{array}{l}\text { 9. Implicó actividades prácticas que sirvieron para } \\
\text { complementar las exposiciones teóricas }\end{array}$ & 30.5 & 51.2 & 12.2 & 4.9 & 1.2 & 4.05 & .86 \\
\hline $\begin{array}{l}\text { 10. El material bibliográfico ofrecido abordó los temas en } \\
\text { profundidad }\end{array}$ & 26.5 & 59 & 9.6 & 2.4 & 2.4 & 4.05 & .83 \\
\hline 11. El material fue de fácil lectura & 42.7 & 48.8 & 3.7 & 2.4 & 2.4 & 4.27 & .85 \\
\hline $\begin{array}{l}\text { 12. Los disertantes explicaron el programa en forma clara } \\
\text { y fácil de entender }\end{array}$ & 36.1 & 48.2 & 10.8 & 2.4 & 2.4 & 4.13 & .88 \\
\hline 13. Los expositores aportaron ejemplos útiles y pertinentes & 39 & 52.4 & 3.7 & 2.4 & 2.4 & 4.23 & .84 \\
\hline 14. El tiempo estuvo bien distribuido & 25.3 & 57.8 & 13.3 & 1.2 & 2.4 & 4.02 & .81 \\
\hline
\end{tabular}

Nota: TA (Totalmente de acuerdo); A (Acuerdo); N (Neutro); D (En Desacuerdo); TD (Totalmente en desacuerdo).

La alta valoración positiva también se ve reflejada en los promedios obtenidos en cada enunciado del cuestionario. En general, dada la escala de 1 a 5 puntos (donde 5 indica la mayor valoración positiva), los ítemes obtuvieron promedios entre 3,95 y 4,33 puntos. El ítem con menor puntaje fue: Brindó herramientas concretas para distinguir cuáles son los niños con problemas asociados a la pobreza en sus clases; y los ítemes valorados más positivamente fueron: Aumentó mi interés en el tema y El material bibliográfico fue de fácil lectura (Ver Tabla 1).

También se solicitó a los participantes que evaluaran la utilidad práctica de esta capacitación en una escala del 1 al 10 (donde 1 correspondía al mínimo de utilidad, y 10 al máximo). El promedio obtenido fue de $8.80(D E=1,18)$. Además, se preguntó a los docentes si recomendaría a sus colegas participar de esta actividad formativa, encontrando que el $90,4 \%$ de las respuestas fueron afirmativas.

Con relación al objetivo 4: Formar a los participantes como agentes multiplicadores para que transfieran al resto del plantel educativo de la institución a la que 
pertenecen, las propuestas generadas; debemos decir que el efecto expansivo es difícil de evaluar, y requiere de un estricto seguimiento de la actividad desarrollada por cada docente que ha participado del programa. No obstante, hemos podido observar que los maestros que han cambiado de grado o de escuela, están actualmente colaborando en la guía continua de sus pares para la implementación de estrategias que promueven la resiliencia.

\section{Conclusiones}

Como producto de la capacitación hemos observado que los docentes lograron detectar y verbalizar sus limitaciones y fortalezas con relación a la formación integral de niños en situación de riesgo por pobreza extrema, adquiriendo herramientas para consolidar las potencialidades y reforzar los puntos débiles.

A través de las actividades grupales realizadas en el marco de cada encuentro, se observó un aumento del interés y de la habilidad para innovar las tareas pedagógicas con el fin de integrar los conocimientos recientemente incorporados. En este sentido, se consideran cumplidos los objetivos básicos de esta propuesta de capacitación.

Las opiniones docentes apoyan estas observaciones, indicando que en general, más del $80 \%$ se considera ahora mejor preparado para enfrentar su tarea áulica cotidiana.

Estos resultados cobran especial relevancia si se tiene en cuenta que en un trabajo anterior, del que participaron 66 docentes de esta misma muestra, el 70,9\% de los maestros afirmó que pocas veces o nunca se incluyen las problemáticas de los niños socialmente vulnerables en el curriculum del magisterio, y el 77,2\% afirmó que durante la carrera docente pocas veces o nunca se infunde capacitación o vocación por el trabajo con los niños en riesgo (Oros, Ghiglione y Menghi, 2010).

Uno de los aspectos más valorados por los maestros tiene que ver con el mayor interés que ha despertado esta capacitación sobre el tema de la pobreza y su impacto sobre el aprendizaje y el bienestar de los alumnos. El interés es uno de los principales motivadores intrínsecos de la conducta, especialmente asociado al crecimiento personal y al esfuerzo creativo, ya que invita a incrementar el conocimiento y la experiencia con el objeto que estimula el interés (Fredrickson, 1998). Siguiendo esta línea, el mayor interés de los docentes por este tema podría desembocar en una mayor probabilidad de innovar las prácticas educativas tradicionales en pos de un mejor acercamiento afectivo y profesional a los niños pobres.

Otro aspecto muy bien valorado tuvo que ver con las características del material bibliográfico ofrecido. Strasser (2006) menciona que es habitual que los investigadores produzcan sus estudios en un lenguaje académico, altamente especializado, haciendo que se vean como poco accesibles o poco relevantes para el trabajo diario de los educadores. Por ello, se considera un logro importante que el material de lectura haya sido identificado como útil y fácil de comprender.

La menor valoración positiva, aunque igualmente alta, se observó en el ítem: Brindó herramientas concretas para distinguir cuáles son los niños con problemas asociados a la pobreza en sus clases. Si bien la situación general de pobreza aumenta la probabilidad de que los niños se encuentren en situación de riesgo psicosocial, cada niño vive una realidad cotidiana particular que determina su propio perfil de 
fortalezas y vulnerabilidades. Dentro de un mismo contexto marginal hay niños que provienen de hogares contenedores y niños que proceden de familias altamente disfuncionales y abandónicas. Quizá una de las tareas más complejas consista en comprender que el término pobreza implica una gran variedad de situaciones, con efecto acumulativo y potenciador, que pueden poner en peligro la salud y el bienestar de los niños en muy diferentes dominios.

Para poder establecer el perfil de riesgo de un niño es necesario tomar en consideración una amplia cantidad de factores simultáneamente. En términos generales se considera que un niño está en riesgo ambiental si sus experiencias de vida están sustancialmente limitadas por la pobreza durante los años de la niñez temprana, con relación al apego desarrollado con sus cuidadores, la organización familiar, el cuidado de la salud, la nutrición y las oportunidades de estimulación física y social (Epps y Jackson, 2000).

Aunque es posible enunciar algunos criterios para reconocer a aquellos niños que manifiestan problemas asociados a la pobreza, poder determinar cuáles son los que presentan más limitaciones a causa de esta situación y cuáles tienen un mejor pronóstico, es bastante más complejo de lo que parece a primera vista y, desde luego, requiere de una evaluación mucho más profunda que la simple apreciación personal del docente y de una lista de criterios concretos enunciados arbitrariamente. Es preciso realizar un análisis contextual integrativo de la realidad de cada niño, tomando en consideración su historia de vida, sus rasgos de personalidad, sus experiencias actuales de convivencia familiar, su estado nutricional y de salud, sus expectativas de cambio, su integración social, su relación con el docente, sus competencias intelectuales, etc. Esta difícil tarea fue presentada a los docentes como uno de los grandes desafíos de la práctica psicológica.

Si bien los resultados discutidos hasta aquí han resultado altamente favorables, corresponde aclarar que el análisis de la eficacia de un programa de capacitación no se agota con la simple opinión de los participantes. Es importante observar si efectivamente se producen cambios en la posterior práctica docente y si ésta a su vez genera un impacto significativo en el desempeño escolar y en la experiencia afectiva y social de los alumnos. Es por ello que durante los últimos años hemos venido reuniendo evidencia científica de cómo estas instancias de capacitación influyen sobre la vocación y el quehacer profesional docente, pero especialmente, sobre el aprendizaje y el bienestar psicosocial de los niños.

En estudios anteriores hemos destacado el impacto que esta propuesta de trabajo ha tenido sobre la experiencia de emociones positivas (Oros, 2009), las habilidades sociales (López e Iglesia, 2007; Musso, López e Iglesia, 2007), las características de personalidad (Lemos, 2007), las funciones ejecutivas (Arán Filippetti y Musso 2007; Arán Filippetti, 2009), las creencias de autoeficacia (Ghiglione 2007), y el desempeño general en la escuela (Richaud de Minzi, 2007) de los niños en situación de riesgo por pobreza.

En síntesis, concluimos que existen suficientes evidencias para afirmar que el ejercicio de la docencia en medios socialmente vulnerables se ve fuertemente beneficiado por la implementación de estas instancias de capacitación docente. Si bien esta tarea no está exenta de dificultades y obstáculos, resulta por demás desafiante. 
"Atreverse a innovar, a dar respuestas creativas, a caminar en la incertidumbre con el norte puesto en la calidad, tal vez despierte deseos en el otro, los refuerce o haga surgir sus potencialidades adormiladas. Valorar los logros y acompañarlo en sus cavilaciones... sin duda, es un desafío interesante para todo capacitador". (Huberman, 2005: 170).

\section{REFERENCIAS BIBLIOGRÁFICAS}

ARÁN FILIPPETTI, V. (2009). Relación entre la planificación, la impulsividad cognitiva y las habilidades intelectuales en niños en riesgo y sin riesgo por pobreza. En M. C. Richaud y J. E. Moreno (Eds.), Investigación en Ciencias del Comportamiento. Avances Iberoamericanos, Tomo I (pp. 33-53). Buenos Aires: Ediciones CIIPME-CONICET.

ARÁN FILIPPETTI, V. y MUSSO, M. (2007). Screening Neuropsicológico en una población de niños bajo riesgo por pobreza. En M. C. Richaud y M. Ison (Eds.), Avances en Investigación en Ciencias del Comportamiento en Argentina: Vol 1, Niñez en riesgo por pobreza (pp. 177-205). Mendoza: Editorial de la Universidad del Aconcagua.

ARÁN FILIPPETTI, V. y RICHAUD DE MINZI, M. C. (2011). Efectos de un programa de intervención para aumentar la reflexividad y la planificación en un ámbito escolar de alto riesgo por pobreza. Universitas Psychologica, 10, 341-354.

ARDILA, R. (2012). ¿Habrá algún día una sociedad sin clases? La compleja relación entre habilidad cognitiva general y nivel socioeconómico. Universitas Psychologica, 11 (2), 663-667.

BRADLEY, R. H.; WHITESIDE, L.; MUNDFROM, D. J.; CASEY, P. H.; KELLEHER, K. J. y POPE, S. K. (1994). Early indications of resilience and their relation to experiences in the home environments of low birthweight, premature children living in poverty. Child Development, 65, 346-360.

BOLZÁN, A. y MERCER, R. (2009). Seguridad alimentaria y retardo crónico del crecimiento en niños pobres del norte argentino. Archivos Argentinos de Pediatría, 107 (3), 221-228.

CEPAL (Comisión Económica Para América Latina y el Caribe) (2009). Panorama social de América Latina. Santigo de Chile: Naciones Unidas.

CIDADE, E. C.; MOURA Jr, J. F. y XIMENES, V. M. (2012). Implicações psicológicas da pobreza na vida do povo latino-americano. Psicologia Argumento, 309680, 87-98.

EPPS, S. y JACKSON, B. J. (2000). Early childhood programs: Models and practices. Washington, DC: American Psychological Association.

FREDRICKSON, B. L. (1998). What good are positive emotions?. Review of general psychology, 2 (3), 300.

GALINDO, O. y ARDILA, R. (2012). Psychology and poverty: Influence of locus of control, self-efficacy and learned helplessness. Avances en Psicología Latinoamericana, 30 (2), 381-407.

GASPARINI, L. y CRUCES, G. (2010). Las asignaciones universales por hijo en Argentina. Impacto, discusión y alternativas. Económica, vol. LVI, 105-146. 
GARRET, P.; NG'ANDU, N. y FERRON, J. (1994). Poverty Experiences of Young Children and the Quality of Their Home Environments. Child Development, 65, 331-345.

GHIGLIONE, M. E. (2007). Percepción de Autoeficacia y Logro Académico en niños frente al fracaso reiterado. En M. C. Richaud y M. Ison (Eds.), Avances en Investigación en Ciencias del Comportamiento en Argentina: Vol. 1, Niñez en riesgo por pobreza (pp. 319-337). Mendoza: Editorial de la Universidad del Aconcagua.

HENDERSON, N. y MILSTEIN, M. M. (2003). Resiliencia en la escuela. Barcelona: Paidós.

HUBERMAN, S. (2005). Cómo se forman los capacitadores: arte y saberes de su profesión. Buenos Aires: Paidós.

LACUNZA, A. B. (2010). Procesamiento cognitivo y déficit nutricional de niños en contextos de pobreza. Psicología y Salud, 20 (1), 77-88.

LEMOS, V. (2007). La personalidad infantil como moduladora del impacto de un proyecto de intervención dirigido a la promoción de resiliencia en niños en riesgo por pobreza. En M. C. Richaud y M. Ison (Eds.), Avances en Investigación en Ciencias del Comportamiento en Argentina: Vol. 1, Niñez en riesgo por pobreza (pp. 259-286). Mendoza: Editorial de la Universidad del Aconcagua.

LÓPEZ, M. y IGLESIA, F. (2007). Conductas asertivas y no asertivas en niños en riesgo por pobreza. Trabajo presentado en las XIV Jornadas de Investigación y Tercer Encuentro de Investigadores en Psicología del MERCOSUR. Publicación de Trabajo completo en Memorias de las XIV Jornadas de Investigación, Tomo II (pp. 530-553).

LOZOFF, B.; JIMENEZ, E.; HAGEN, J.; MOLLEN, E. y WOLF A. W. (2000). Poorer behavioral and developmental outcome more than 10 years after treatment for iron deficiency in infancy. Pediatrics, 105 (4), e51. Disponible en: http://pediatrics. aappublications.org.

LUPIEN, S. J.; KING, S.; MEANEY, M. J. y McEWEN, B. S. (2001). Can poverty get under your skin? Basal cortisol levels and cognitive function in children from low and high socioeconomic status. Development and psychopathology, 13 (03), 653-676.

McLOYD, V. C. y WILSON, L. (1991). The strain of living poor: Parenting, social support, and child mental health. En A. C. Huston (Ed.), Children in poverty: Child development and public policy (pp. 105-135). Nueva York: Cambridge University Press.

MCLANAHAN, S. S.; ASTONE, N. M. y MARKS, N. (1991). The role of mother-only families in reproducing poverty. En A. C. Huston (Ed.), Children in poverty: Child development and public policy (pp. 51-78). Nueva York: Cambridge University Press.

MILMAN, N. (2012). Fisiopatología e impacto de la deficiencia de hierro y la anemia en las mujeres gestantes y en los recién nacidos/infantes. Revista Peruana de Ginecología y Obstetricia, 58 (4), 293-312.

MUSSO, M. F.; LÓPEZ, M. e IGLESIA, F. (2007). Auto-Regulación y habilidades Sociales: Intervención en una población infantil en riesgo por pobreza. En M. C. Richaud y M. Ison (Eds.), Avances en Investigación en Ciencias del Comporta- 
miento en Argentina: Vol. 1, Niñez en riesgo por pobreza (pp. 209-236). Mendoza: Editorial de la Universidad del Aconcagua.

OROS, L. B. (2009). Impacto de una intervención continua para estimular las emociones positivas en niños y niñas afectados por la pobreza. En M. C. Richaud y J. E. Moreno (Eds.), Investigación en Ciencias del Comportamiento. Avances Iberoamericanos, Tomo I (pp. 243-255). Buenos Aires: Ediciones CIIPME-CONICET.

OROS L. B.; GHIGLIONE, M. E. y MENGHI, M. S. (2010). Una propuesta de capacitación docente para optimizar el aprendizaje en contextos de pobreza. Memorias de XVII Jornadas de Investigación. Sexto Encuentro de Investigadores en Psicología del MERCOSUR, “Clínica e Investigación. Contribución a las Problemáticas Sociales". Tomo I. Buenos Aires: UBA - Facultad de Psicología, 514.

OROS, L. B.; MANUCCI, V. y RICHAUD DE MINZI, M. C. (2011). Desarrollo de emociones positivas en la niñez. Lineamientos para la intervención escolar. Revista Educación y Educadores, 14 (3), 493-509.

PAREDES, Y. (2013). Desnutrición, cognición y pobreza. Revista UNIMAR, 31 (2), 69-79. PATEL, V. y KLEINMAN, A. (2003). Poverty and common mental disorders in developing countries. Bulletin of the World Health Organization, 81 (8), 609-615.

RAMEY, C. T. y CAMPBELL, F. A. (1991). Poverty, early childhood education, and academic competence: The Abecedarian experience. En A. C. Huston (Ed.), Children in poverty: Child development and public policy. Nueva York: Cambridge University Press.

RICHAUD, M. C. (2013). Contributions to the study and promotion of resilience in socially vulnerable children. American Psychologist, 68 (8), 751-758. doi: 10.1037/a0034327. Disponible en: http://www.ncbi.nlm.nih.gov/pubmed/24320672.

RICHAUD, M. C.; MESTRE, M. V.; LEMOS, V.; TUR, A.; GHIGLIONE, M. y SAMPER, P. (2013). La influencia de la cultura en los estilos parentales en contextos de vulnerabilidad social. Avances en Psicología Latinoamericana, 31 (2).

RICHAUD DE MINZI, M. C. (2007). Fortalecimiento de recursos cognitivos, afectivos, sociales y lingüísticos en niñez en riesgo ambiental por pobreza: Un programa de intervención. En M. C. Richaud y M. Ison (Eds.), Avances en investigación en Ciencias del Comportamiento en Argentina: Vol. 1, Niñez en riesgo por pobreza (pp. 147-176). Mendoza: Editorial de la Universidad del Aconcagua.

RICHAUD DE MINZI, M. C. (2009). Sin afecto no se aprende ni se crece. Un programa para reforzar los recursos afectivos, cognitivos y lingüísticos en niños en riesgo por pobreza extrema. Boletín SIP-Argentina. La Psicología en Argentina, 10. RICHAUD DE MINZI, M. C.; SACCHI, J. C. y MORENO, J. E. (2003). Sin afecto no se aprende ni se crece: Un programa para fortalecer los recursos afectivos, cognitivos y lingüísticos en niños en riesgo por extrema pobreza. (Inédito) Proyecto PICT (14064).

SINGER, L. T.; MINNES, S.; SHORT, E.; ARENDT, R.; FARKAS, K.; LEWIS, B.; KLEIN, N.; RUSS, S.; MIN, M. y KIRCHNER, L. (2004). Cognitive Outcomes of Preschool Children with Prenatal Cocaine Exposure. JAMA, 291 (20), 2448-2456. Disponible en: http://jama.jamanetwork.com/journal.aspx. 
SMITH, D. C. y SANDHU, D. S. (2004). Toward a Positive Perspective on Violence Prevention in Schools: Building Connections. Journal of Counseling and Development, 82, 287-293.

SOLANO, L.; ACUÑA, I.; BARÓN, M. A.; MORÓN DE SALIM, A. y SÁNCHEZ, A. (2008). Influencia de las parasitosis intestinales y otros antecedentes infecciosos sobre el estado nutricional antropométrico de niños en situación de pobreza. Parasitología latinoamericana, 63 (1-2-3-4), 12-19.

STANCO, G. G. (2007). Funcionamiento intelectual y rendimiento escolar en niños con anemia y deficiencia de hierro. Colombia Medica; 38 (1), 24-33. Disponible en: http://hdl.handle.net/10893/4687.

STRASSER, K. (2006). Evaluación de programas de intervención temprana. Revista En Foco, 78.

WILSON, W. J. (1996). When Work Disappears: The World of the New Urban Poor. Nueva York: Knopf. 